\title{
Respiratory Distress in SARS-CoV-2 without Lung Damage: Phrenic Paralysis Should Be Considered in COVID-19 Infection
}

\author{
Francois Maurier, Benoit Godbert, Julie Perrin
}

Hôpitaux Privés de Metz, Metz, France

Received: $14 / 05 / 2020$

Accepted: $14 / 05 / 2020$

Published: $21 / 05 / 2020$

\begin{abstract}
How to cite this article: Maurier F, Godbert B, Perrin J. Respiratory distress in SARS-CoV-2 without lung damage: phrenic paralysis should be considered in
\end{abstract} COVID-19 infection. EJCRIM 2020;7: doi:10.12890/2020_001728.

Conflicts of Interests: The Authors declare that there are no competing interests.

This article is licensed under a Commons Attribution Non-Commercial 4.0 License

\section{ABSTRACT}

We describe an overweight COVID-19 patient with respiratory distress preceded by anosmia/dysgeusia with no lung injury shown on CT, angio-CT or ventilation/perfusion scans. Orthopnoea and paradoxical abdominal respiration were identified. Phrenic paralysis, demonstrated by examination of patient breathing, and on $x$-ray while standing breathing in and out, explained the respiratory distress. This is a rare and previously undescribed neurological complication of COVID-19 infection caused by vagus nerve injury.

\section{LEARNING POINTS}

- Phrenic paralysis must be kept in mind as a rare neurological complication of COVID-19.

- Vagus nerve palsy is a neurological manifestation as anosmia and dysgeusia, that were already identified in the olfactory system of COVID-19 patients.

\section{KEYWORDS}

COVID-19, respiratory distress, phrenic paralysis, neurotropism

\section{INTRODUCTION}

The COVID-19 outbreak started in China and was first described as a common but serious lung infection with fever, cough and pneumonia. Other clinical manifestations were noted in Europe, indicating SARS-CoV-2 infection was a systemic and multifaceted disease with neurological, cardiovascular and digestive symptoms and complications due to acute and diffuse endothelial dysfunction, which could also induce a dysregulated and overwhelming cytokine storm. Initially ignored, the peripheral and central neurological complications may explain some clinical manifestations such as vagus nerve palsy.

\section{CASE DESCRIPTION}

A never-smoking 58-year-old woman, with no significant medical history except for obesity (BMI $33 \mathrm{~kg} / \mathrm{m}^{2}$ ), complained of fever, dysgeusia, anosmia and rapidly progressive dyspnoea on 16 March 2020. An RT-PCR test for COVID-19 was positive. A chest CT scan was normal. On 24 March, dyspnoea at rest, fever and oxygen saturation of $88 \%$ in room air were noted. The patient refused hospitalization and remained at home in bed. Her clinical situation did not improve. On 3 April, CT angiography of the chest and a ventilation/perfusion scan were performed but did not show pulmonary embolism. Parenchyma remained normal. D-dimers, NT-pro-BNP and cardiac echocardiography were normal.

On 30 April, the patient consulted a pulmonologist in our clinic. Dyspnoea was improving but was still very severe, occurring with minimal effort. She complained of a dry cough and did not describe any haemoptysis. She had orthopnoea and paradoxical abdominal respiration in 
the supine position was observed. A pure restrictive pulmonary syndrome was noted with a forced vital capacity (FVC) of 1.28 litre (46\%) and forced expiratory volume in 1 second (FEV1) of 1.1 litre (47\%). The FEV1/FVC ratio was $86 \%$. FVC worsened by more than $20 \%$ in the supine position to 0.98 litre (30\% of theoretical value). We were unable to perform plethysmography or a gas transfer test for carbon monoxide (TLCO) because the patient was too tired and breathless.

Chest x-rays were taken in complete inspiration and expiration (Fig. 1). The inspiration and expiration images were very similar, while the rib cage in profile does not expand during inspiration.

Ultrasound of the diaphragm was not informative because of obesity. Electromyography of the diaphragm was not possible due to the patient's location as in France this test is only available in Paris. Creatinine phosphate kinase levels were normal.

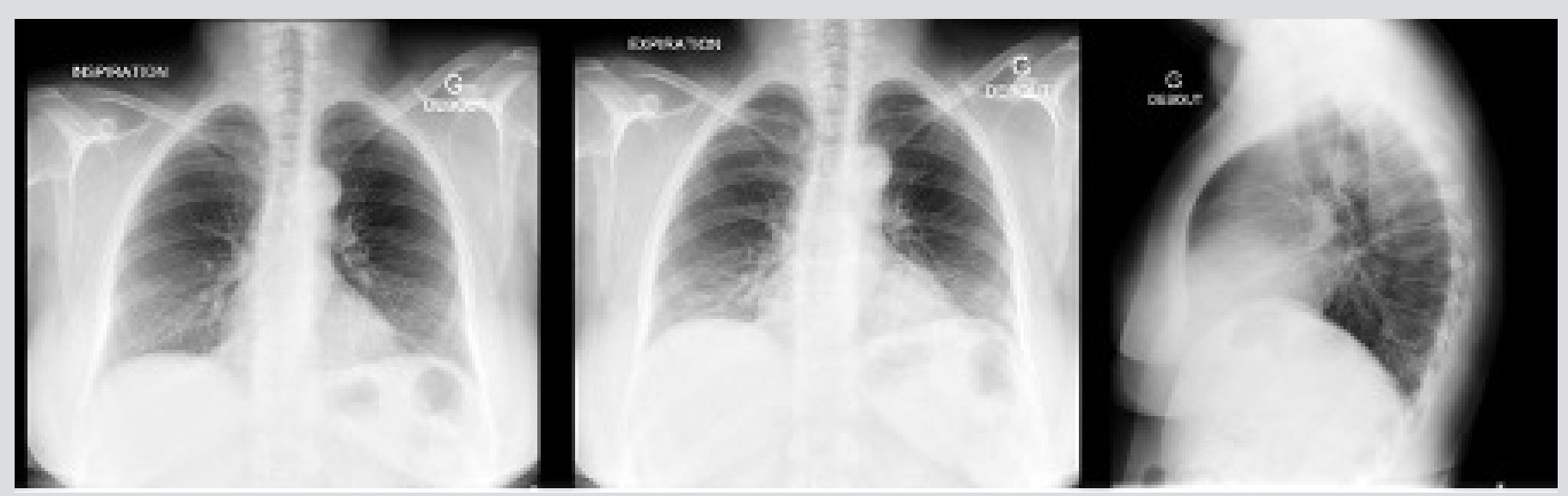

Figure 1. Chest $x$-ray: the inspiration and expiration images are almost the same. The rib cage in profile does not expand during inspiration

\section{DISCUSSION}

We have described a patient with phrenic paralysis inducing pulmonary failure without any cardiac, pleural, parenchymal or vascular pulmonary abnormalities. We suggest this was caused by direct peripheral neurological involvement of phrenic nerves ${ }^{[1]}$. Virologists have described neurological lesions causing anosmia and dysgeusia ${ }^{[2]}$, which were not recognized in patients in Wuhan ${ }^{[3]}$. Anosmia and dysgeusia were thought to result from direct viral attack on olfactory nerve cells. Mononeuritis multiplex ${ }^{[4]}$, meningoencephalitis, myelitis and vigilance disorders have been described as a result of SARS-CoV-19 neurotropism. Increasing evidence also shows that SARS-CoV-19 may invade peripheral nerve terminals ${ }^{[5]}$. This observation suggests the virus may cause phrenic paralysis in COVID-19 patients, resulting in pulmonary decompensation.

\section{CONCLUSIONS}

Phrenic paralysis should be considered in COVID-19 lung infection when orthopnoea and paradoxical abdominal respiration are present without vascular or parenchymal abnormalities on thoracic CT scan. The possibility of neurological assault by SARS-CoV-2 on peripheral nerves, especially the vagus nerve, should be determined by investigation. Its association with anosmia and dysgeusia, another neurological symptom, should be considered.

\section{REFERENCES}

1. Matsuda K, Park CH, Sunden $\mathrm{Y}$, Kimura T, Ochiai K, Kida H, et al. The vagus nerve is one route of transneural invasion for intranasally inoculated influenza A virus in mice. Vet Pathol 2004;41(2):101-107.

2. Desforges M, Le Coupanec A, Dubeau P, Bourgouin A, Lajoie L, Dubé M, et al. Human coronaviruses and other respiratory viruses: underestimated opportunistic pathogens of the central nervous system? Viruses 2019;12(1).

3. Chen T, Wu D, Chen H, Yan W, Yang D, Chen G, et al. Clinical characteristics of 113 deceased patients with coronavirus disease 2019: retrospective study. BMJ 2020;368:m1091.

4. Zhao H, Shen D, Zhou H, Liu J, Chen S. Guillain-Barre syndrome associated with SARS-CoV-2 infection: causality or coincidence? Lancet Neurol 2020;19(5):383-384.

5. Li YC, Bai WZ, Hashikawa T. The neuroinvasive potential of SARS-CoV2 may play a role in the respiratory failure of COVID-19 patients. J Med Virol 2020;92:552-555. 